\title{
Knowledge Fusion and Synchronization over Ubiquitous Ontology Mapping
}

\author{
Hang Qin', Li Zhu ${ }^{2 *}$ \\ ${ }^{1}$ Computer School, Yangtze University, Jingzhou, China \\ ${ }^{2}$ Oujiang College, Wenzhou University, Wenzhou, China \\ Email: `68681700@qq.com, ${ }^{\star}$ yeah_1397118@hotmail.com
}

How to cite this paper: Qin, H. and $\mathrm{Zhu}$, L. (2017) Knowledge Fusion and Synchronization over Ubiquitous Ontology Mapping. Journal of Computer and Communications, 5, 1-9.

https://doi.org/10.4236/jcc.2017.510001

Received: July 1, 2017

Accepted: July 27, 2017

Published: July 30, 2017

Copyright (C) 2017 by authors and Scientific Research Publishing Inc. This work is licensed under the Creative Commons Attribution International License (CC BY 4.0).

http://creativecommons.org/licenses/by/4.0/

\section{Open Access}

\begin{abstract}
With the rapid development and popularization of web services, the available information types and structure are becoming more and more complex and challenging. Actually web services involve the need for dynamic integration and transparent knowledge integration, in light of the urgent information changing track. Under this situation, the traditional search engine and information integration cannot finish this challenge, thereby bringing the opportunity for knowledge fusion and synchronization. This paper proposes a multi-matching strategy ontology mapping method for web information, i.e., ubiquitous ontology mapping method (U-Mapping), which can be viewed as a base collection of information on multiple ontologies made to appear anytime and everywhere. This approach is usually built independently by different information providers, avoiding the grammatical and semantic conflict. Finally, the ontology case information can be utilized under the consolidation of the U-Mapping, concerning language technology and machine learning methods.
\end{abstract}

\section{Keywords}

Ubiquitous Ontology Mapping, Knowledge Fusion, Information Integration, Knowledge Synchronization, Conceptual Space, Fusion Rules

\section{Introduction}

Ubiquitous computing is a concept in computer science and software engineering, where computing is made to appear anytime and everywhere. In contrast to desktop computing, ubiquitous computing can occur using any device, in any location, and in any format. In terms of ambient intelligence, the information source is often to build a collection of information communicating with each other over web technologies [1] [2]. Due to the independent autonomy of in- 
formation sources, the ontologies made by different people, are a set of concepts and categories in a subject area or domain, which shows the properties and the relations between them [3] [4]. To achieve information sharing and communication purposes, it is the ontology that can establish semantic mapping with entities interrelationships that really or fundamentally exist for a particular domain of discourse. In this paper, we build the ontology mappings for urgent need of ubiquitous computing. The corresponding semi-automatic or automatic completion of the mapping process can save a lot of favor [5] [6]. Consequently, we propose a ubiquitous ontology mapping method (U-Mapping) under a multimapping strategy.

As the most important manner to get information, web scale is growing at a rapid rate. However, the vast majority of the information on the Internet is represented in a readable format, e.g., HTML, and the software program and software agent cannot understand or deal with these information, concerning the potential far from being excavated [7] [8]. In order to facilitate comparison with the method of existence, the conceptual space will be replaced by the ontology to study related issues.

With the development of the semantic web, the information is gradually expressed in a semi-structured and structured form, and the ontology is used to show the semantics. Ontology is an explicit description of the conceptual world, which allows the knowledge of the domain to be expressed as a concept class system, where each concept has its own attributes [9] [10]. So, the software agent can be related to the body to understand the meaning of information, thereby completing the web information collection and integration automatically.

However, different information sources may be represented by different ontologies, and there is no single ontology to do all the work [11]. In this paper, in order to achieve the information exchange, it is necessary to establish semantic mapping. Also, in the semantic web, information sources are more and more, and these information sources can establish a set of instances on the ontology. Furthermore, due to the independent autonomy of the information source, the ontologies can be built by different people, thereby having semantic conflict due to ontological diversity.

Because the ontology development is different, the corresponding modeling method used is different. There is many a discrepancy in the ontology developed by experts among different fields, such as the use of different terms and concepts. This paper builds the ubiquitous ontology mapping, which is to find the semantic links of the concepts, thus achieving the purpose of sharing multiple sources of information.

\section{Background}

There are many automatic mode or semi-automatic ontology mapping tools, and most tools are implemented the simplest (1:1) mapping. According to the classification method, these tools can be attributed to two categories: 
1) The ontology information mapping tool is mainly based on ontology information, while the ontology structure is to determine the similarity of the concept. They are compared with the concept name to produce a match list, while the user has the mapping list to determine the final concept mapping.

2) Instance-based ontology mapping is concerned with ontology information over the instances to determine the mapping relationship. They are analyzed by the examples to calculate the concept of similarity, and do not consider the concept of the relationship.

Ontology mapping issues play an important role in many applications, such as semantic Web, schema/ontology integration, data warehouse, XML message mapping, etc. Typically, building ontology mapping is an important part and prerequisite of information integration system.

Once the mapping is established, it is possible consolidate ontology by merging algorithm. In addition to the above applications, ontology mapping plays an important role in many system interoperability and proxy exchange. Specifically, we propose a new ubiquitous ontology mapping method (U-Mapping) architecture in Figure 1, with its evolution, domain knowledge and constraints, cooperative consensus building, and GUI. In addition, there are different programming languages, such as Python, Java, and others.

How to automatically or automatically achieve ontology mapping is still an important challenge. To achieve a fully automated ontology mapping, which is difficult, especially for some complex matches ( $n: m)$.

\section{Problem Formulation}

Ontology research is related to pattern matching, which are data members joined by certain data structures. The collection is a logical-level view of a data member. Most works are in a certain application environment and specific patterns and so on. Currently, the main study focuses on how to achieve automatic/semiautomatic matching of patterns. Thus, we have the following definitions:

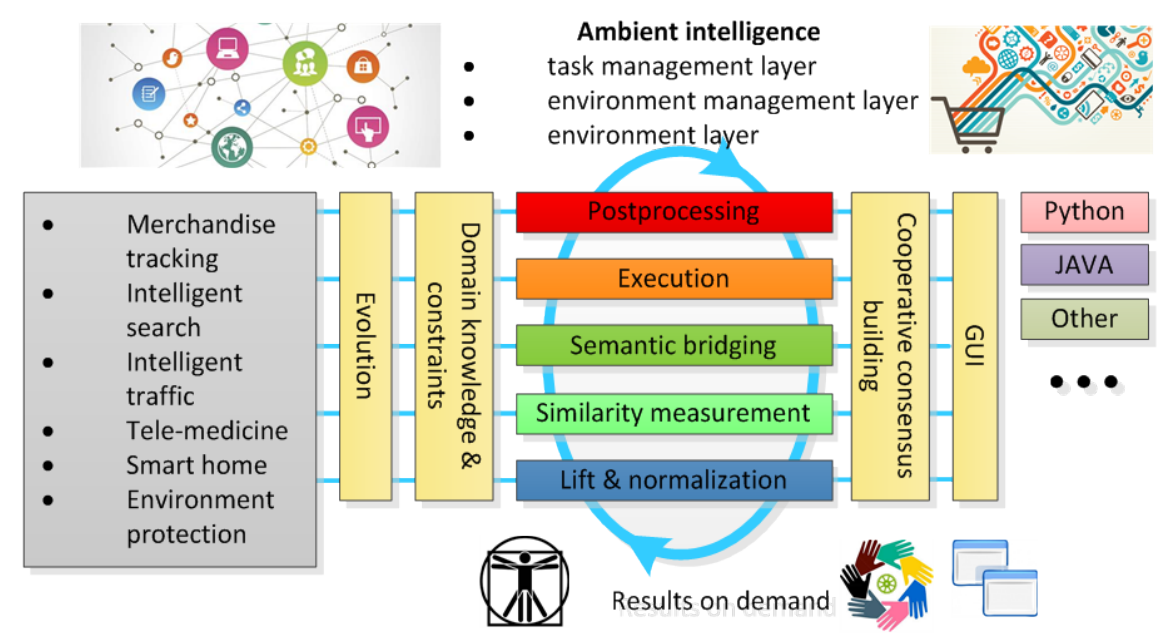

Figure 1. The U-Mapping architecture over devices connected to various applications. 


\subsection{Ubiquitous Ontology Mapping}

Definition 1 (ontology mapping) Given two ontologies $O_{1}, O_{2}$, and an application on both ontologies. If there are ontological elements $e_{1} \in O_{1}, e_{2} \in O_{2}$, the results $P\left(e_{1}\right)$ and $P\left(e_{2}\right)$ for $P$ are equal or similar, there are semantic correspondence between $e_{1}$ and $e_{2}$, denoted as $M\left(e_{1}\right)=e_{2}$. This semantic matching process is called ontology mapping.

This type of mapping is a (1:1) mapping relationship. In addition, there are (1:n), (n:1), (n:m) mapping relationship.

Definition 2 (ontology merge/integration) Given two ontologies $O_{1}, O_{2}$, according to the two ontologies expressed by semantic system, to produce a new ontology $O^{\prime}$ process.

The semantic similarity function of the ontology element is consistent with the object similarity with the following properties:

1) $\operatorname{Sim}(x, y) \in[0,1]$, where $x$ and $y$ are the ontology elements.

2) $\operatorname{Sim}(x, y)=1$, where two ontologies are semantically identical.

3) $\operatorname{Sim}(x, y)=0$, where two ontologies have completely different semantics.

4) $\operatorname{Sim}(x, x)=1$, where similarity satisfies reflexivity.

5) $\operatorname{Sim}(x, y)=\operatorname{Sim}(y, x)$, where the similarity satisfies symmetry.

In general, in the ontology mapping process, the similarity calculation of the ontology elements is a sufficient condition to judge the mapping between ontology elements. The similarity of the ontology element is to return a value between 0 and 1.

\subsection{Mapping Description}

Because the developer of the ontology is different, the modeling method used must be different. As for the same domain problem modeling, different areas of experts to develop the body must exist. Before the discussion for the mapping, we first give a formal definition of ontology mapping problem:

Definition 3 Given two ontologies $O_{1}, O_{2}$, the mapping problem can be defined as a 5-tuple $\left(O_{1}, O_{2}, M_{c}, M_{a}, M_{r}\right)$.

1) The mapping $M_{c}: C_{O_{1}} \rightarrow C_{O_{2}}$, if there exists $c \in C_{O_{1}}, c^{\prime} \in C_{O_{2}}$, satisfying $\operatorname{Sim}\left(c, c^{\prime}\right)$ is greater than the given threshold, then the two concepts are considered semantically the same.

2) The mapping $M_{a}: A_{O_{1}} \rightarrow A_{O_{2}}$, if $a \in A_{O_{1}}, a^{\prime} \in A_{O_{2}}$, satisfying $\operatorname{Sim}\left(a, a^{\prime}\right)$ is greater than the given threshold, it is assumed that the two attribute slots are semantically the same.

3) The mapping $M_{r}: R_{\mathrm{O}_{1}} \rightarrow R_{\mathrm{O}_{2}}$, if there is $r \in R_{\mathrm{O}_{1}}, r^{\prime} \in R_{\mathrm{O}_{2}}$, satisfying $\operatorname{Sim}\left(r, r^{\prime}\right)$ is greater than a given threshold, the two troughs are considered semantically the same.

The ontology mapping is mainly considered with the concept and the mapping between the two aspects, the two aspects can affect each other. It is assumed that there is a global ontology. The purpose of mapping is for a new addition plus the information source, to find out the information on the body and the 
overall ontology mapping.

The process of semantic mapping is to find the process of the concept in $O_{1}$ that corresponds to the semantics of a concept of pattern $\mathrm{O}_{2}$. Such as the concept in $O_{1}$ and $O_{2}$. Consequently, there is a semantic correspondence between the results on demand.

\section{Ubiquitous Ontology Mapping Method}

\subsection{Mapping Classification}

The integration of heterogeneous data sources has always been an important topic. Ontology relative to the database model deals with the semantics of its richer, larger scale. There are still many similarities in their mapping studies.

For ontology mapping, a multi-strategy approach is generally used. In the specific implementation, the general need to combine a variety of matching methods, according to the specific application requirements to flexibly use different methods combined. For a separate matching, we consider the following irrelevant classification criteria: based on pattern and based on instance, the former only considers the model information, regardless of the instance. Pattern information includes name, description, relationship (part-of, is-a, etc.) and constraints.

Element-level matching considerations are a single concept, attribute, and relationship in the ontology. Consider the logical link between concepts, i.e., it does not take into account when matching the parent concept its sub-concept and the relationship between other concepts. In contrast, structural level matching not only takes into account individual objects. Structure may be exactly matched or may be partial matching, depending on the required completeness and accuracy of the match. Ideally, ontology in the same structure with all the concepts can be one by one correspondence.

The most commonly used information in language-based methods is element names and textual descriptions. There are many similarities in measuring the name, such as equivalence, synonymy, upper and lower relationships, editing distance and so on. In the specific applications, domain-related dictionaries contain domain knowledge, is particularly helpful. Constraint information refers to attribute and relationship definition information, including the value of the domain, uniqueness, potential and so on. If the comparison method has such constraint information, it is used to determine the similarity of the pattern elements.

The relationship over cardinal matching is divided into local and global. If we consider in different mapping rules, that is global. Most of the existing matching methods are associated with an element in an ontology pattern over the highest similarity in another ontology pattern.

\subsection{Consolidation of Multiple Methods}

Each matching method takes advantage of different information. For a given 
matching task, each method has its own applicability and characteristics. Thus, the combination of several single methods tends to produce better results than in one way. There are two general combinations: Hybrid matching is the use of multiple matching criteria to complete the pattern/ontology element matching, while combined matching refers to the fact that each single matcher alone completes the pattern/ontology element matching, merging on the results.

The hybrid matching method can use multiple criteria throughout the process, and provide more good candidate results and performance. This is because it is in the early stages of a standard result of the exclusion. The results of the methods are combined, and these methods can also include the mixing method, so it has greater flexibility.

The selection of the matching method, the execution of the decision and the merging of the independent run results, both of which can be matched by the matching method itself automatic decision. The automated approach can reduce the participation, but it is difficult to get fit common solutions for different application areas, although they can be controlled by adjusting parameters. In any case, the user's participation is essential, because itself only provides some candidate results, and requires the user to decide whether to receive or reject ultimately.

In order to handle complex matching tasks, the matching process should be an iterative development process, which always supports multiple users. In the combination method, each matching method can be executed in a certain order, and the matching result provided by the user can also be used as an independent matching algorithm. For the matching input by the user, the combination matching method must be aware of its authority, so the focus on solving the mismatch part.

\section{Ubiquitous Process with Similarity}

\subsection{Similarity}

Ontology mapping is determined by ontology element similarity. In this paper, the result of each matching method is a reflection (or element match pairs), each mapping element is assigned a value of 0 (completely different) to 1 (exactly the same), which indicates the degree of similarity between the corresponding elements. Here, we can pay attention to one-to-one (1:1) matching results. The matching algorithm gives a plurality of ontology elements the matching result with different similarity values, and then the final decision result is determined by the user, including the matching result of (n:m) case. As for the knowledge, the $\mathrm{U}$ Mapping architecture is shown in Figure 2, from input source, database ontology mapping, knowledge application framework, to user interface and application, considering web service and phone service.

There are four parts in the knowledge application framework, i.e., recommendation system, decision support system, question answering system, and semantic search system, in light of file transport of diverse target resources, i.e., 


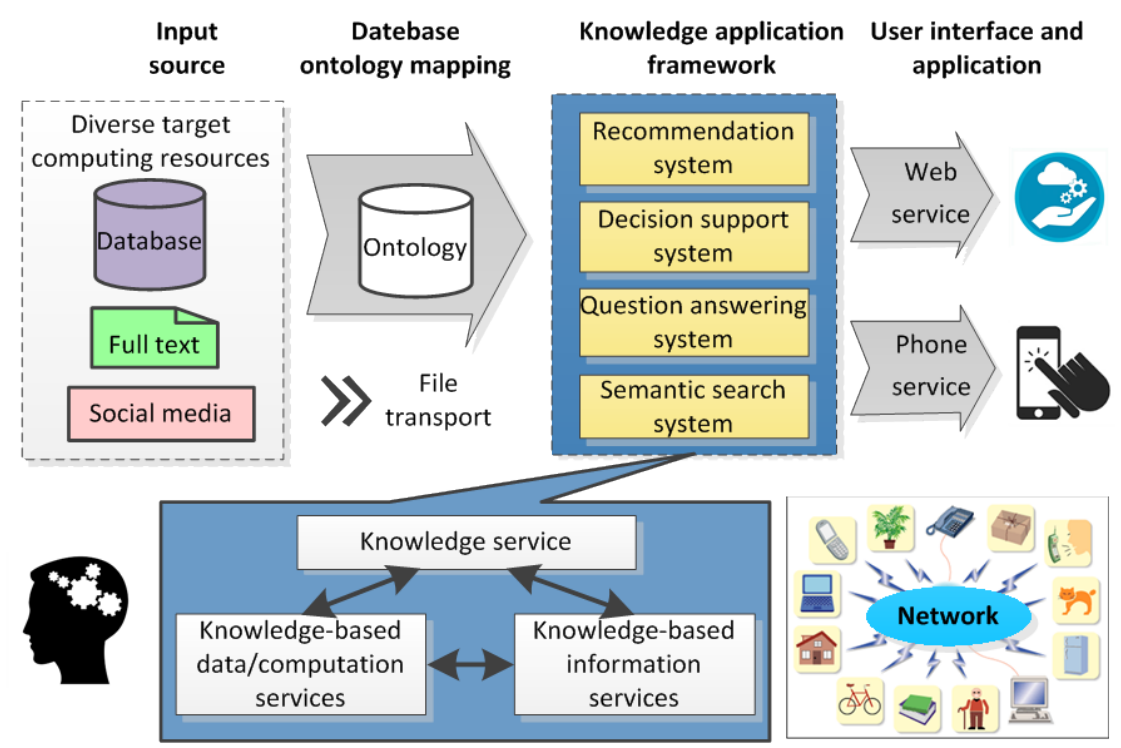

Figure 2. U-Mapping with knowledge fusion being available anywhere.

database, full text, and social media. The ontology elements are a set of simple, meaningful strings to identify. In general, the same concept may be represented by a different name, and the same name may indicate a different meaning. However, with the same domain relationship, the comparison element name is still feasible.

Conceptual similarity refers to the definition of semantic relations between concepts from two different ones. The name of the concept represents the semantics of the concept, but because of the length and difficulty of abstraction, it often cannot accurately reflect the concept. Therefore, in the conceptual matching method based on ontology information, the similarity of concepts is mainly determined by attribute similarity. In addition, relationships can also determine the concepts to a certain extent.

This is because each instance of the information source is an instance of a leaf concept. If every leaf concept in the source ontology can find an instance, it is also a target ontology example of leaf concepts. However, the same instance information is used to determine whether the ontology element is not because finding the same instance can lead to a dramatic increase in the complexity of space and time. On one hand, if the two instances do not have the same instance, the matching result may not be obtained to determine the semantic correspondence of elements. On the other hand, the relationship between the concepts in the ontology is defined by the implication and synonyms between the concepts. The semantics of the concept are determined by the description of the attribute set.

\subsection{Mapping Rules}

In order to better define the conceptual matching relationship in the ontology, we defines four matching guidance rules, which can be used in the database on- 
tology mapping process in Figure 2.

Rule 1 If the properties of the two concepts are completely comparable, i.e., the two classes have a common set of attributes and the attributes are true value, the range is related, then the two classes are synonymous, denoted as $C_{1} \equiv C_{2}$.

Rule 2 If the properties of the two concepts are partially comparable, i.e., a class $\left(c_{1}\right)$ an a class $\left(c_{2}\right)$ on all the attributes are true, and the range is related, vice versa is not established, then called the package including the relationship, denoted as $c_{2} \supseteq c_{1}$.

Rule 3 If the properties of the two concepts are not comparable, i.e., an instance of class $\left(c_{1}\right)$ is in another class $\left(c_{2}\right)$ on all the attributes will not have value, vice versa is not established, then called the concept does not have a semantic relationship.

Rule 4 If the two concepts have the same direct subordinate concept, the two concepts are considered synonymous. If the two concepts have a similar direct upper concept, and the concept of the same name, that the two concepts are synonymous.

\section{Conclusion}

In summary, we propose the ubiquitous ontology mapping method U-Mapping with knowledge fusion, synchronization, and similar semantic vocabulary. Also, we can effectively deal with the concept of matching with the learning method, with regard to the concept of the connotation of a set of attributes. Moreover, the application of synonym dictionaries can effectively address some semantic similar but spelling different lexical mappings. Finally, under the similarity analysis and mapping rules, the semi-automatic ontology mapping method of multimapping strategy can utilize the combining and mixing method to identify the complex matching algorithm.

\section{References}

[1] Anam, S., Kim, Y.S., Kang, B.H., et al. (2016) Adapting a Knowledge-Based Schema Matching System for Ontology Mapping. Proceedings of the Australasian Computer Science Week Multiconference, Canberra, 1-5 February 2016, 27. https://doi.org/10.1145/2843043.2843048

[2] Laporte, M.A., Valette, L., Cooper, L., et al. (2016) Comparison of Ontology Mapping Techniques to Map Plant Trait Ontologies. Joint International Conference on Biological Ontology and BioCreative (2016), Corvallis, 1-4 August 2016.

[3] Kumar, S.K. and Harding, J.A. (2013) Ontology Mapping Using Description Logic and Bridging Axioms. Computers in Industry, 64, 19-28. https://doi.org/10.1016/j.compind.2012.09.004

[4] Arch-int, N. and Arch-int, S. (2013) Semantic Ontology Mapping for Interoperability of Learning Resource Systems Using a Rule-Based Reasoning Approach. Expert Systems with Applications, 40, 7428-7443. https://doi.org/10.1016/j.eswa.2013.07.027

[5] Jung, J.J. (2013) Semantic Wiki-Based Knowledge Management System by Interleaving Ontology Mapping Tool. International Journal of Software Engineering and 
Knowledge Engineering, 23, 51-63. https://doi.org/10.1142/S0218194013400044

[6] Fang, A., Hong, N., Wu, S., et al. (2013) An Integrated Biomedical Ontology Mapping Strategy Based on Multiple Mapping Methods. The 14th International Conference on Web Information System Engineering, Nanjing, 13-15 October 2013, 373386.

[7] Senthil Kumaran, V. and Sankar, A. (2013) Towards an Automated System for Intelligent Screening of Candidates for Recruitment Using Ontology Mapping (EXPERT). International Journal of Metadata, Semantics and Ontologies, 8, 56-64. https://doi.org/10.1504/IJMSO.2013.054184

[8] Gao, W. and Zhu, L. (2014) Gradient Learning Algorithms for Ontology Computing. Computational Intelligence and Neuroscience, 2014, Article ID: 438291. https://doi.org/10.1155/2014/438291

[9] Bevilacqua, M., Tsourdos, A., Starr, A., et al. (2015) Data Fusion Strategy for Precise Vehicle Location for Intelligent Self-Aware Maintenance Systems. 2015 6th IEEE International Conference on Intelligent Systems, Modelling and Simulation (ISMS), Kuala Lumpur, 9-12 February 2015, 76-81. https://doi.org/10.1109/ISMS.2015.37

[10] Vieira, G.G., Varela, L.R. and Ribeiro, R.A. (2016) A Knowledge Based System for Supporting Sustainable Industrial Management in a Clothes Manufacturing Company Based on a Data Fusion Model. 2016 International Conference on Decision Support System Technology, Plymouth, 23-25 May 2016, 113-126.

[11] Campbell, M.E. and Ahmed, N.R. (2016) Distributed Data Fusion: Neighbors, Rumors, and the Art of Collective Knowledge. IEEE Control Systems, 36, 83-109. https://doi.org/10.1109/MCS.2016.2558444

Submit or recommend next manuscript to SCIRP and we will provide best service for you:

Accepting pre-submission inquiries through Email, Facebook, LinkedIn, Twitter, etc. A wide selection of journals (inclusive of 9 subjects, more than 200 journals)

Providing 24-hour high-quality service

User-friendly online submission system

Fair and swift peer-review system

Efficient typesetting and proofreading procedure

Display of the result of downloads and visits, as well as the number of cited articles

Maximum dissemination of your research work

Submit your manuscript at: http://papersubmission.scirp.org/

Or contact jcc@scirp.org 